\title{
Accuracy and Repeatability of Joint Centre Location in Computer-Assisted Knee Surgery
}

\author{
Kevin B. Inkpen and Antony J. Hodgson \\ Department of Mechanical Engineering \\ University of British Columbia, Vancouver, BC, Canada
}

\begin{abstract}
To properly align knee prostheses, we must accurately define the mechanical axis which joins the hip and ankle centres. Current computerassisted techniques rely on markers pinned not only to the distal femur and proximal tibia, but also to the pelvis and calcaneus, thereby increasing pain and the risk of infection. To eliminate these pins remote from the knee, we are designing non-invasive "trackers" that are strapped to the patient's pelvis and foot and are insensitive to skin motion. We mounted our prototype trackers on a fresh cadaver alongside conventional bone pin markers in the pelvis and calcaneus, and located the hip and ankle centres 30 times. We then dissected out and digitized the femoral head. Results from the hip tracker were excellent, the mean centre being within $0.2 \mathrm{~mm}$ (ML) and $1.3 \mathrm{~mm}$ (AP) of the physical centre of the femoral head (all results at $95 \%$ confidence limit). Compared to the digitized centre, the hip tracker introduces mechanical axis error of less than $0.07^{\circ}$ (frontal plane) and $0.25^{\circ}$ (sagittal plane) $95 \%$ of the time, while the pelvic bone pin introduces $0.03^{\circ}$ (frontal) and $0.14^{\circ}$ (sagittal) error. Results were not as good at the ankle: although there was no significant difference in mean ankle centre location between the foot tracker and the calcaneus bone pin $(\mathrm{P}=0.09$ in $\mathrm{ML}, \mathrm{P}=0.08$ in $\mathrm{AP})$, both means differed from the anatomical ankle centre (digitized midpoint between malleoli) by $5-7 \mathrm{~mm}$, a mechanical axis difference of $\sim 0.5^{\circ}$. Compared to the digitized mechanical axis, overall axis definition will be within $1.2^{\circ}$ (frontal) and $0.9^{\circ}$ (sagittal) using bone pins and $1.3^{\circ}$ (frontal) and $2.0^{\circ}$ (sagittal) using non-invasive trackers $95 \%$ of the time. For both methods, almost all error arises from bias and lack of precision at the ankle. We conclude that bone pins at the hip are unnecessary for subdegree accuracy, but that better methods of locating the ankle centre should be investigated.
\end{abstract}

\section{Introduction}

We are developing computer assisted total knee replacement (TKR) tooling that eliminates intramedullary rods and improves alignment accuracy without introducing additional imaging requirements (such as preoperative CT scans) or invasive procedures (such as bone pins remote to the operating site). Current non-computer assisted techniques have overall limb alignment standard deviations (SD) of $2.5^{\circ}$ to $3.0^{\circ}([1,2,3])$. Since alignment errors of as little as $3^{\circ}$ have been shown to cause 
poor outcomes [4] and normal asymptomatic knees are typically within $1^{\circ}$ of neutral alignment [5], our goal is to achieve a SD of $1^{\circ}$; this improvement will be large enough to potentially improve outcomes and will enable us to assess the effect of alignment on implant longevity. Prosthesis alignment begins with an accurate definition of the mechanical axis (the line between the hip and ankle centres) since this axis is the datum to which bone cuts and the resulting alignment are referenced. Additional errors are introduced when positioning the knee centre and joint line relative to the mechanical axis and when making the bone cuts, so we must be able to define the mechanical axis to a SD well within $1^{\circ}$ in order to reach our overall alignment accuracy goal.

The computer assisted TKR technique developed by Leitner [6] defines the mechanical axis intraoperatively using a 3D optoelectronic localizer. They find the hip centre by tracking a point at the distal femur in a reference frame rigidly pinned to the pelvis as they move the femur through its range of motion. Similarly, they find the ankle centre by tracking a marker pinned to the talus or calcaneus in a coordinate frame rigidly pinned to the tibia. In contrast to conventional TKR, this technique requires two incisions and bone pin holes remote from the operating site which increases the patient's pain and risk of infection. In an effort to eliminate bone pins remote from the operating site, we have designed and tested devices that are strapped to the patient intraoperatively and used to track the motion of the pelvis and the foot without requiring incisions or immobilization. In this paper we compare the accuracy and repeatability of mechanical axis definition using our prototype non-invasive hip and foot trackers to results using bone pins, and we compare both methods to the mechanical axis defined by digitizing the femoral head and ankle centre.

\section{Methods}

\subsection{Hip and Foot Tracker Design}

In order to minimize artifacts due to relative motion caused by skin sliding over bone, we designed our hip and foot trackers to constrain all six degrees of freedom by applying six reaction forces only normal to the bone (see Figure 1; note that proximaldistal components of forces are not shown). The trackers are fully adjustable to accommodate different patients and seating forces are applied by straps. Each tracker has an optical marker array rigidly attached. 


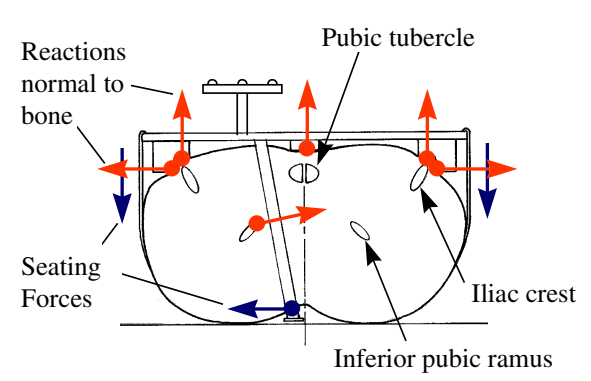

(a) Transverse section (looking proximally) through pelvis

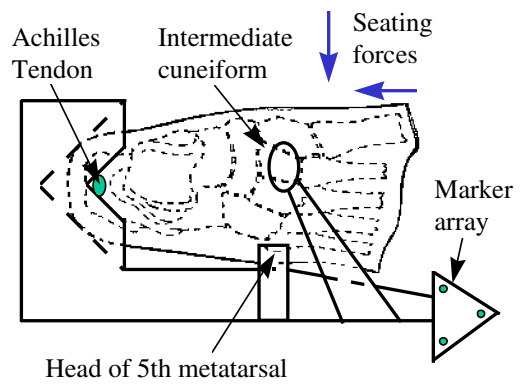

(b) View looking distally on right foot.

Fig. 1. Hip tracker (a) and foot tracker (b) schemes

\subsection{Setup and Procedure}

We adjusted the hip and foot trackers and strapped them to a fresh cadaver (female, 89 y.o., $1.45 \mathrm{~m}$ tall, $760 \mathrm{~mm}$ hip-ankle). We drove bone pins (4 $\mathrm{mm}$ dia) into the ilium, distal femur, proximal tibia, and calcaneus on the right side, and rigidly attached one array of three infrared emitting diodes (IREDs) to each bone pin.

We used a Flashpoint 5000 localizer (Image Guided Technologies, Boulder, CO, USA), which has a typical accuracy of $\sim 0.5 \mathrm{~mm}$ [7] to track the markers. We found that its noise level (SD of reported IRED position under static conditions) was $\sim 0.12$ $\mathrm{mm}$ at the typical poses in our study. For digitizing, we used the $135 \mathrm{~mm}$ two-emitter point probe supplied with the localizer. Each coordinate frame is defined by an array of 3 IREDs arranged in an equilateral triangle, $120 \mathrm{~mm}$ on a side for the pelvis and hip tracker and $60 \mathrm{~mm}$ on a side at the femur, tibia, calcaneus, and foot tracker. We recorded the transforms between all the coordinate frames in anatomic position, with a flat surface pressed against the soles of the feet to approximate the ground plane. We then digitized the distal tips of the malleoli and defined the midpoint between them as the anatomic ankle centre.

To find the hip centre, we recorded 50 points from a single IRED on the distal femur array as the femur was moved slowly through the maximum range of motion possible, creating a 'cloud' of data points in the pelvic bone pin coordinate frame. At each of the 50 sampling points, we also recorded the pose of the hip tracker marker array. We found the hip centre in pelvic bone pin coordinates by fitting a sphere (by least squares) to the data and also found the corresponding centre in hip tracker coordinates by expressing each data point in hip tracker coordinates using the homogeneous transform recorded at that point, fitting a sphere, and transforming the resulting centre back to pelvic pin coordinates using the anatomic position transform. We repeated this procedure 30 times, producing 30 pairs of hip centres. Similarly, we 
calculated 30 ankle centres in the tibial coordinates by tracking one IRED each on the foot tracker and calcaneus pin arrays. All resulting centres are in tibial coordinates and can be compared directly. We also recorded the poses of the foot tracker and calcaneus arrays at each sampling point so that we could detect their relative motions.

\subsection{Data Analysis}

All centres are expressed in coordinates aligned with the body axes (ML = medial/lateral, AP $=$ anterior/posterior, $\mathrm{PD}=$ proximal/distal $)$. We consider the digitized results to be the control and compare them to each of the two treatments (trackers and bone pins) separately to find maximum expected errors. To compare the bone pins to the trackers, we analyze the differences between them in each direction under the null hypothesis that the mean difference is zero. All groups of 30 centres and differences were normally distributed (checked using normal scores plots), so we used standard t-tests. Based on the variance of the differences in each direction, we can detect differences between the bone pins and the trackers of $0.25 \mathrm{~mm}$ (ML) and $0.76 \mathrm{~mm}(\mathrm{AP})$ at the hip and $4.7 \mathrm{~mm}(\mathrm{ML})$ and $5.2 \mathrm{~mm}(\mathrm{AP})$ at the ankle $95 \%$ of the time (95\% power).

The angular error in defining the mechanical axis is proportional to the joint centre location errors and inversely proportional to the mechanical axis length. To be conservative, we have used the mechanical axis length $(750 \mathrm{~mm})$ for a $5^{\text {th }}$ percentile female (mean of Japanese and Swedish populations; $~ 1.5 \mathrm{~m}$ tall) for computing all angular errors.

To portray the motion of the trackers relative to the bone pin frames, we compute changes in the vector between the nominal joint centre and the origin of the tracker coordinate frame as the limb is moved; we plot these changes on a $5^{\circ}$ grid representing the limb motions (e.g., flexion \& abduction); each motion vector represents the average motion in the surrounding $5^{\circ} \times 5^{\circ}$ neighbourhood and the magnitude will depend on the location of the tracker coordinate frame.

\section{Results}

Figures $2 \& 3$ show average range of motion across the 30 trials, digitized joint centres, and the resulting groups of centres from both the trackers and the bone pins. Frontal plane motion of the hip tracker is shown in Fig. 4. Motion plots of the hip tracker in the sagittal plane and the foot tracker are not shown due to space limitations. Relative to the ilium pin coordinate frame, the mean magnitude of movement of the hip tracker coordinate origin was $3.2 \mathrm{~mm}$ (range $2.0 \mathrm{~mm}$ to $3.8 \mathrm{~mm}$ ). Relative to the calcaneus pin coordinate frame, the mean magnitude of movement of the foot tracker coordinate origin was $22 \mathrm{~mm}$ (range $14 \mathrm{~mm}$ to $26 \mathrm{~mm}$ ). 


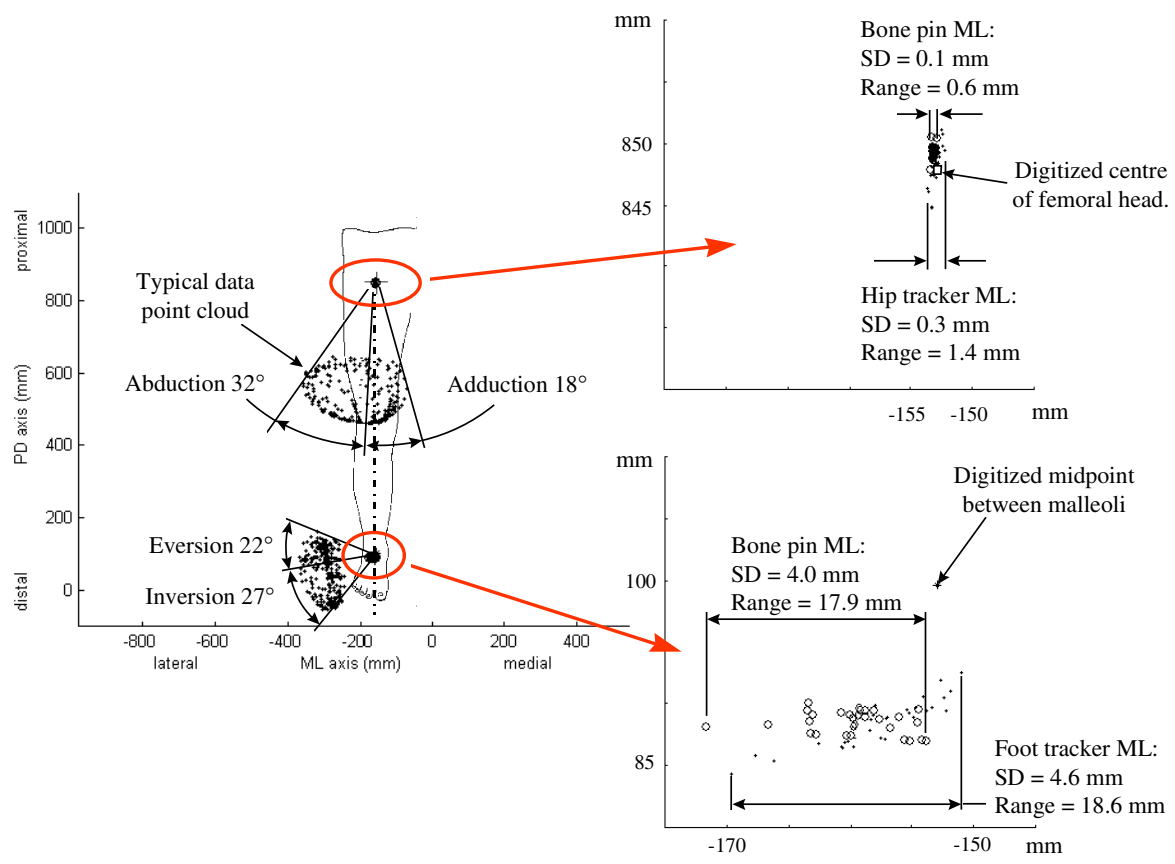

Fig. 2. Frontal plane view, mean centres and ranges of motion, 30 trials

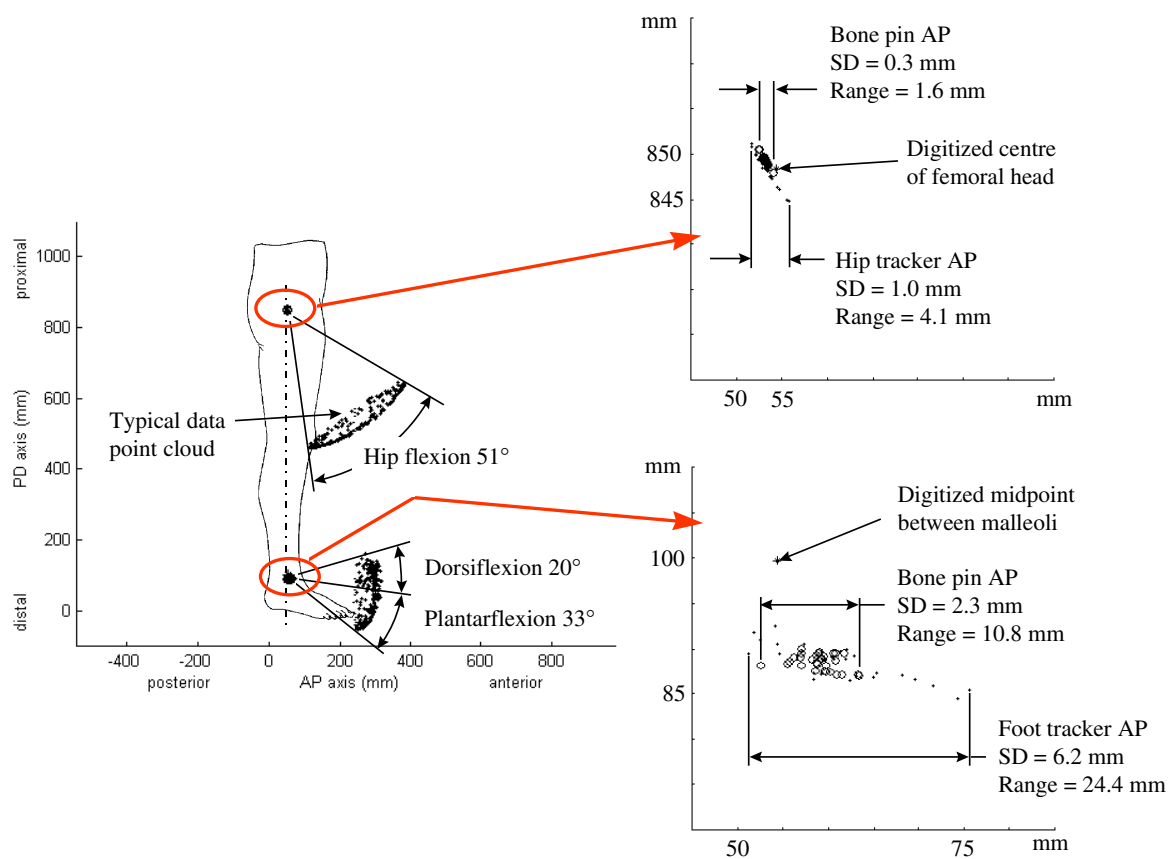

Fig. 3. Sagittal plane view, mean centres and ranges of motion, 30 trials. 


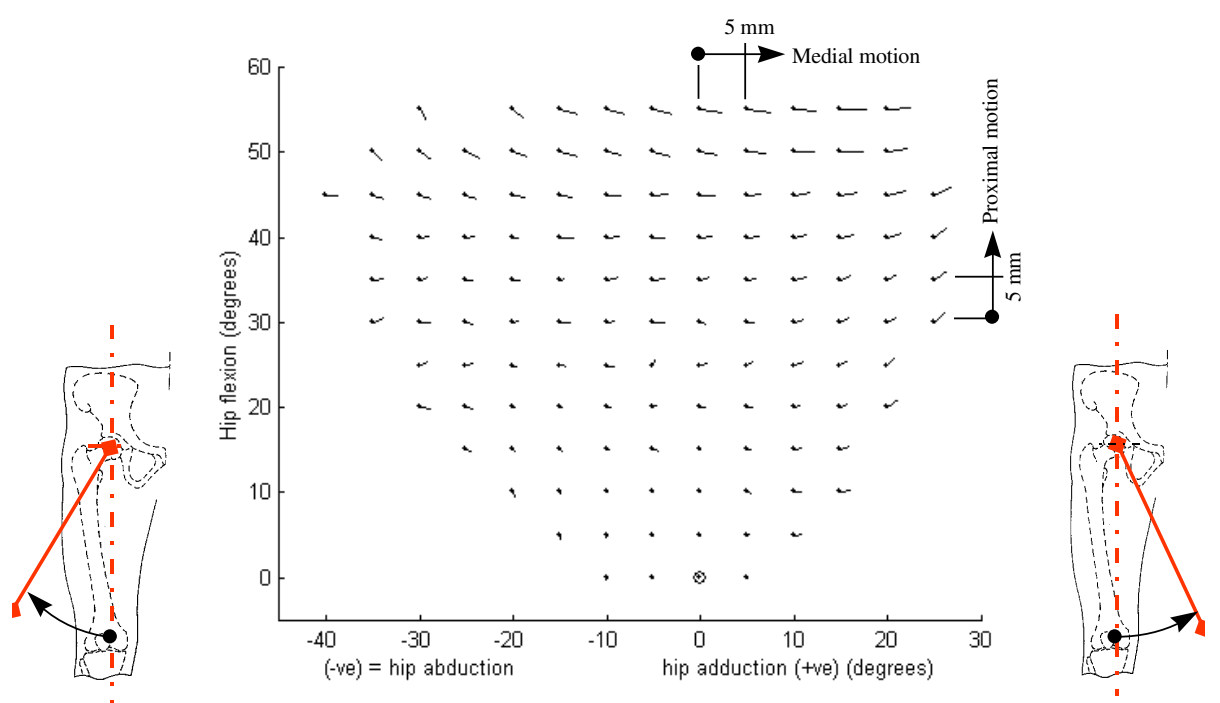

Fig. 4. Motion of hip tracker in frontal plane vs. femur position, mean of 30 trials

\section{Discussion}

The mean hip centre found using the hip tracker is $0.09 \mathrm{~mm}$ medial (95\% CI -0.03 to $0.21, \mathrm{P}=0.14), 0.94 \mathrm{~mm}$ posterior $(95 \% \mathrm{CI} 0.58$ to $1.3, \mathrm{P}<0.001)$, and $0.11 \mathrm{~mm}$ distal $(95 \% \mathrm{CI}-0.43$ to $0.66, \mathrm{P}=0.67$ ) to the digitized femoral head centre. Mechanical axis definition using the pelvic bone pin will be within $0.03^{\circ}$ (frontal plane) and $0.14^{\circ}$ (sagittal plane) of the digitized result $95 \%$ of the time (at $95 \%$ confidence). Although the hip tracker results are $\sim 3 \mathrm{x}$ more variable, the corresponding mechanical axis errors are still very low at less than $0.07^{\circ}$ (frontal) and $0.25^{\circ}$ (sagittal) $95 \%$ of the time. Compared to the pelvic bone pin, the mean hip centre found with the hip tracker was $0.23 \mathrm{~mm}$ medial (95\% CI 0.10 to 0.35 , $\mathrm{P}<0.001)$ and $0.17 \mathrm{~mm}$ anterior $(95 \% \mathrm{CI}-0.21$ to $0.55, \mathrm{P}=0.37)$. In $\mathrm{PD}$, which has little effect on the mechanical axis definition, the tracker mean was $1.1 \mathrm{~mm}$ distal (95\% CI 0.51 to $1.7, \mathrm{P}<0.001$ ). The motion of the hip tracker relative to the ilium is comparable to motion found by Lea [8] for a pelvis immobilizer. The directions of motion are as expected, with a medial shift particularly in flexion and adduction due to the thigh contacting the rod passing through the crotch. Results using the hip tracker may be worse for obese patients, and some bruising may occur at the iliac crests; we are therefore continuing to test and refine our hip tracker.

At the ankle, both the foot tracker and bone pin methods are highly variable and produce mean centres distinctly different from the digitized ankle centre (midpoint between the distal tips of the malleoli). The calcaneus bone pin mean is $6.7 \mathrm{~mm}$ lateral, $4.7 \mathrm{~mm}$ anterior, and $11 \mathrm{~mm}$ distal to the digitized point, resulting in a mean $0.51^{\circ}$ abduction and $0.36^{\circ}$ flexion difference in mechanical axis definition. The 
foot tracker mean is $4.6 \mathrm{~mm}$ lateral, $7.0 \mathrm{~mm}$ anterior, and $11 \mathrm{~mm}$ distal to the digitized point, resulting in $0.35^{\circ}$ abduction and $0.54^{\circ}$ flexion. Mechanical axis definition using the calcaneus bone pin will be within $1.26^{\circ}$ (frontal plane) and $0.80^{\circ}$ (sagittal plane) of the digitized result $95 \%$ of the time (at $95 \%$ confidence). The corresponding errors using the foot tracker are within $1.23^{\circ}$ (frontal) and $1.72^{\circ}$ (sagittal). Note that compared to the bone pin, the mean ankle centre found with the foot tracker was not significantly different at $2.0 \mathrm{~mm}$ medial $(95 \% \mathrm{CI}$. -0.31 to $4.39, \mathrm{P}=0.09), 2.4 \mathrm{~mm}$ anterior $(95 \% \mathrm{CI}-0.29$ to $4.98, \mathrm{P}=0.08)$, and $0.13 \mathrm{~mm}$ distal $(95 \% \mathrm{CI}-0.74$ to 0.47 , $\mathrm{P}=0.65)$. The foot tracker was significantly less precise in the AP $(\mathrm{P}<0.001)$ and $\mathrm{PD}(\mathrm{P}<0.001)$ directions but ML precision was not significantly different $(\mathrm{P}=0.21)$.

We would expect some difference between digitized and motion tracking results at the ankle because they are really two different measurements: The digitized ankle centre roughly corresponds with the centre of rotation of the talus alone [9], making the mechanical axis pass roughly through the centre of area of the talo-crural joint, while the calcaneus bone pin and the foot tracker motions include the effects of the subtalar joint, with its externally rotated and inverted axis of rotation. Therefore, by using calcaneus motion tracking and sphere fitting at the ankle, we are modelling a biaxial joint complex as a spherical joint. In pilot studies, we have found tracking a bone pin in the talus to be the most imprecise method, particularly in the frontal plane (as expected due to limited inversion/eversion). Considering that virtually all existing studies and techniques of lower limb alignment refer to the midpoint between the malleoli, we consider the digitized ankle centre to be the current 'gold standard'. The current results suggest that either an explicitly biaxial representation of the ankle joint complex or a robust method of digitizing the ankle centre should be investigated as an alternative to fitting a sphere to tracked motions of a bone pin or a foot tracker.

As found in [10], we expect the main source of alignment error in a passive guidance system to be the implementation. Bone cutting errors of up to $1^{\circ}$ are expected with current techniques [11], and error will also be introduced in finding the appropriate references at the knee [12]. Mechanical axis definition error arises from the sum of the variances and biases at the hip and ankle, so we can see from the current study that the ankle centre definition contributes almost all of the error. As implementation errors will add to registration error, the current results show that even if bone pins are used at the ankle, it will be difficult to achieve sub-degree accuracy in final limb alignment.

\section{Conclusions}

We conclude that for sub-degree alignment accuracy in computer assisted knee surgery, bone pins at the hip can be eliminated, but at the ankle a robust method of digitizing the joint centre should be developed as an alternative to motion tracking methods. 


\section{Acknowledgements}

Thanks to Dr. Robert W. McGraw, UBC/Vancouver General Hospital Dept. of Orthopaedics, Dr. Thomas Oxland, UBC Division of Orthopaedic Research, and Dr. Vlad Stanescu, UBC Dept. of Anatomy for their assistance.

\section{References}

1. Elloy MA, Manning MP, Johnson R: Accuracy of Intramedullary Alignment in Total Knee Replacement. Journal of Biomedical Engineering, 14 (5), 1992, pp. 363-370

2. Ishii Y, Ohmori G, Bechtold JE, Gustilo RB: Extramedullary vs. Intramedullary Alignment Guides in Total Knee Arthroplasty. Clinical Orthopaedics (318), 1995, pp. 167175

3. Jessup DE, Worland RL, Clelland C, Arredondo J: Restoration of Limb Alignment in Total Knee Arthroplasty: Evaluation and Methods. J. Southern Orthopaedic Assoc. 6 (1), 1997, pp. 37-47

4. Jeffery RS, Morris RW, Denham RA: Coronal Alignment After Total Knee Replacement. J. of Bone and Joint Surgery (British Ed.) 73-B, 1991, pp. 709-714

5. Nguyen C, Bryant JT, Cooke TDV, Chow D: Alignment and Geometry of the Normal Knee in Stance. J. of Bone and Joint Surgery (British Ed.) 71-B(3) 1989, pp. 346

6. Leitner F, Picard F, Minfelde R, Schulz H-J, Cinquin P, Saragaglia D: Computer Assisted Knee Surgical Total Replacement. In: Troccaz, J., Grimson, E., Mosges, R. (eds): CVRMed-MRCAS '97. Lecture Notes in Computer Science Vol. 1205, Springer-Verlag 1997, pp. 627-638.

7. Chassat F, Lavallee S: Experimental Protocol of Accuracy Evaluation of 6-D Localizers for Computer-Integrated Surgery: Application to Four Optical Localizers. In: Wells, W; Colchester, A; Delp, S. (eds): Medical Imaging and Computer Assisted Intervention (MICCAI'98). Lecture Notes in Computer Science Vol. 1496, Springer-Verlag 1998, pp. 421-430.

8. Lea JT, Mills A, Watkins D, Peshkin M, Kienzle T, Stulberg SD: Registration and Immobilization for Robot-Assisted Orthopaedic Surgery. In: DiGioia, Kanade, Taylor, eds: First International Symposium on Medical Robotics and Computer Assisted Surgery (MRCAS) 1994. Shadyside Hospital, Pittsburgh, PA, USA, pp 63-68.

9. Lundberg A, Svensson OK, Nemeth G, Selvik G: The Axis of Rotation of the Ankle Joint. J. of Bone and Joint Surgery (British Ed.) 71-B, 1989, pp. 94-99

10. Moody JE, DiGioia AM, Jaramaz B, Blackwell M, Colgan B, Nikou C: Gauging Clinical Practice: Surgical Navigation for Total Hip Replacement. In: Wells, W; Colchester, A; Delp, S. (eds): Medical Imaging and Computer Assisted Intervention (MICCAI'98). Lecture Notes in Computer Science Vol. 1496, Springer-Verlag 1998, pp. 421-430.

11. Otani T, Whiteside LA, White SE: Cutting Errors in Preparation of Femoral Components in Total Knee Arthroplasty. Journal of Arthroplasty, Vol 8, No 5, Oct. 1993 pp. 503-510.

12. Inkpen KB, Emrich RJ, Hodgson AJ: Probe Design to Robustly Locate Anatomical Features. In: Wells, W; Colchester, A; Delp, S. (eds): Medical Imaging and Computer Assisted Intervention (MICCAI'98). Lecture Notes in Computer Science Vol. 1496, Springer-Verlag1998,pp.335-342. 